\title{
Are malignant melanoma time trends explained by changes in histopathological criteria for classifying pigmented skin lesions?
}

\author{
ROBIN PHILIPP, 1 ANDREW \\ JENNIFER SIZER ${ }^{3}$ \\ HASTINGS, ${ }^{2}$ \\ JAMES \\ BRIGGS, ${ }^{2}$ \\ AND
}

From the Department of Epidemiology and Community Medicine, ${ }^{1}$ University of Bristol, Department of Pathology, ${ }^{2}$ Frenchay Hospital and Torbay Hospital, ${ }^{3}$ Torquay

SUMMARY The incidence of malignant melanoma in developed countries has been rising rapidly in recent years. Its causes and the reasons for this trend are uncertain. Changes in histopathological criteria for classifying pigmented skin lesions could explain the rising incidence rates. With support from the International Agency for Research on Cancer, this hypothesis is explored but not substantiated for time trends in the South Western Region, United Kingdom.

The incidence of malignant melanoma has been rising in areas of the world for which epidemiological data are available. ${ }^{1}$ For example, in Scandinavia the incidence had been doubling every 10 years, ${ }^{2}$ and in Queensland, Australia, every 15 years. $^{3}$ In New Zealand, for the past 30 years there has been an annual incremental increase of $7.5 \%$ in age-standardised incidence rates. ${ }^{4}$ In England and Wales, mortality from cutaneous malignant melanoma has more than doubled since 1950, and the incidence continues to rise. ${ }^{5}$ Reasons for these time trends and causes of the tumour remain obscure. ${ }^{16}$

One possible explanation for the increasing incidence rates is a change in histopathological criteria for the diagnosis of malignant melanoma. This hypothesis is supported from a large series of malignant melanoma cases assembled by a WHO group in which a definite tendency to include increasingly thinner lesions was noted during the 10 year collection period (IARC, personal communication, 1984). Furthermore, in a series of histological reports gathered between 1958 and 1979 from several large surgical pathology practices in Birmingham, Alabama, USA, many melanocytic lesions originally diagnosed as junctional naevi were found on review to be malignant melanomas in situ. ${ }^{7}$ In 1982, the need for a standard internationally accepted histopathological classification of malignant melanoma was also reported. ${ }^{8}$ More recently, concern has been expressed that world experts in melanoma pathology differ in their opinion concerning the malignant or benign nature of 'borderline' lesions; it has been suggested that the sharply rising incidence rates in parts of the world could be more apparent than real. ${ }^{9}$
Against this background the International Agency for Research on Cancer, World Health Organisation, $\frac{O}{\vec{D}} \vec{A}$ developed an international collaborative study with $\stackrel{\circ}{\circ}$ 으 ten participating pathology departments in $\stackrel{\mathbb{Q}}{\stackrel{1}{2}}$ Scandinavia, Western Europe, and Australasia to $\overline{3}$ determine whether a change in histological 0 interpretation of pigmented skin lesions is discernibleo $\frac{\rho}{\supset}$ within each department with time and, if so, whether geographical differences in interpretation are observed when a sample of histological material is circulated between the collaborating centres. The University of Bristol and Frenchay Health Authority, Bristol, were invited to take part. In this paper we report the findings from a review of histological criteria that have been used since 1945 to classify pigmented skin lesions in the South Western Region, UK.

\section{Method}

In accordance with the protocol prepared by the International Agency for Research on Cancer, three time periods were chosen for study: 1945-49, 1954-57, and 1980 . For each time period, stored paraffin block material for 50 original benign lesions and 20 original malignant lesions was identified by two consultant histopathologists as representative samples from several hundred available lesions. The benign lesions were deliberately chosen to include $\mathbf{4 0}$ junctional or compound naevi for which a change of opinion over time was thought most likely to arise, and 10 intradermal lesions which were considered less likely to be confused with malignant melanoma. Benign lesions comprised diagnoses of naevus, naevus pigmentosum, naevus cellularis, junctional naevus, 
active junctional naevus with early/questionable malignancy, or any synonym or variant of these diagnoses. Likewise, malignant lesions could include synonyms or variants of the diagnosis 'malignant melanoma,' such as superficial spreading melanoma and invasive melanoma.

For each lesion, 15 haematoxylin and eosin slides were prepared. If several paraffin blocks were available for a given lesion, the most representative block was selected. These slides were then examined 'blind' by a histopathologist and reported as benign, ? malignant, early malignant/level $1 /$ in situ, or frankly malignant. More detailed histopathological reporting was not required as the review was intended to study time trends in reporting the 'benign' or 'malignant' nature of lesions and not the congruence between pathologists. The diagnosis on review was then compared with that originally reported.

\section{Results}

As shown in table 1, and among a total of 210 lesions that were studied, four lesions were reclassified to the 'benign' or 'malignant' group on histological review. This proportion of lesions that were reclassified is not statistically different from the proportion (206/210) that were not reclassified to a different group. Likewise, the differences in numbers that were reclassified during each of the three time periods under review are also not statistically significant.

As shown in table 2, more detailed histopathological comment, using guidelines of the International Agency for Research on Cancer protocol and as described in the methodology, resulted in the classification of 188 lesions remaining unchanged. The remaining 22 lesions were reclassified in either a 'more' or a 'less' sinister direction. Nevetheless, these changes were generally minor points of detail. As noted in table 1, only one lesion was consequently repositioned from the 'benign' to the 'malignant' group, and three lesions were placed in the 'benign' from the 'malignant' group. Altogether, there were 11 minor changes for the 1945-49 time period, five for 1954-57 and six for the year 1980. These

Table 1 Histological reclassification* of pigmented skin lesions into 'benign' and 'malignant' groups for three time periods reviewed in 1985

\begin{tabular}{lllll}
\hline Year & Diagnosis & $\begin{array}{l}\text { Total } \\
\text { 'benign' }\end{array}$ & $\begin{array}{l}\text { Total } \\
\text { 'malignant' }\end{array}$ & $\begin{array}{l}\text { McNemar's } \\
\text { test }\end{array}$ \\
\hline $1945-49$ & Original & 50 & 20 & ns \\
& Revised & 51 & 19 & ns \\
$1954-57$ & Original & 50 & 20 & ns \\
& Revised & 52 & 18 & 20 \\
\end{tabular}

\footnotetext{
Classification groups:

1 Benign

2 ? Malignant

3 Early malignant/level $1 /$ in situ

4 Frankly malignant
}

Table 2 Histological reclassification* of pigmented skin lesions for three time periods reviewed in 1985

\begin{tabular}{|c|c|c|c|c|}
\hline \multirow[t]{2}{*}{ Changes in classification } & \multicolumn{3}{|c|}{ Time period } & \multirow[b]{2}{*}{$\begin{array}{l}\chi^{2} \text { test } \\
\text { for trend }\end{array}$} \\
\hline & $1945-49$ & 1954-57 & 1980 & \\
\hline Classification unchanged & 59 & 65 & 64 & ns \\
\hline $\begin{array}{l}\text { Reclassified in more } \\
\text { sinister direction }\end{array}$ & 8 & 2 & 3 & ns \\
\hline $\begin{array}{l}\text { Reclassified in less } \\
\text { sinister direction }\end{array}$ & 3 & 3 & 3 & ns \\
\hline Totals & 70 & 70 & 70 & \\
\hline
\end{tabular}

\footnotetext{
- Classification groups:

1 Benign

2 ? Malignant

3 Early malignant/level $1 /$ in situ

4 Frankly malignant
} 
apparent time trends are, however, statistically insignificant when the $\chi^{2}$ test for trend is applied.

\section{Discussion}

Within the UK, the South Western Region has the highest incidence rates of malignant melanoma in both males and females. ${ }^{10}$ Since 1948, when Frenchay Hospital opened, it has been the major regional referral centre for patients with malignant melanoma. ${ }^{11}$ Concurrently, there has also been considerable interest there for the preoperative diagnostic accuracy of this tumour, ${ }^{12}$ and in its epidemiology, ${ }^{13}$ as well as more general interest in possible approaches to histopathological audit. ${ }^{14}$ Accordingly, although 16\% (11/70) of paraffin blocks from the 1945-49 time period in this study were reclassified in a 'more' or 'less' sinister direction when reviewed in 1985, the changes were minor and resulted in only one lesion being reclassified as 'benign' rather than 'malignant'; fewer lesions were regrouped for each of the two more recent time periods that were also reviewed. As previously noted, none of the differences was statistically significant. The findings of this study are therefore reasssuring for the time trends in histological criteria required for labelling a pigmented skin lesion as 'malignant', and there seems to have been no trend since 1945 for borderline benign or malignant lesions to be differently classified within the South Western Region. Our findings therefore support the worth of Lloyd's classification of melanomas, a system that was used at Frenchay Hospital between 1948 and the late 1970s. ${ }^{11}$ More recently, in the South Western Region, UK, and in support of the need for a widespread standard classification ${ }^{8}$ and concern for appropriate methods of early recognition of thin tumours, ${ }^{15}$ the anatomical site, Breslow thickness, and Clark's level of invasion have been introduced in the regional melanoma reporting system.

In conclusion, we have found no evidence that rising incidence rates for malignant melanoma in the South Western Region, UK, are explained by changes in histopathological criteria for classifying pigmented skin lesions. A similar conclusion was also reached for Northern Ireland, from a study there of 267 possible cases of cutaneous malignant melanoma. ${ }^{16}$ Reasons for the worldwide rising incidence rates of malignant melanoma remain uncertain.

We are very grateful to the International Agency for Research on Cancer, WHO, and the Unit Management Group, Frenchay Health Authority, for encouragement and support to undertake this study as part of the IARC International Collaborative Study of Pigmented Skin Lesions. We are also indebted to the Medical Laboratory Scientific Officers in Pathology Departments of Frenchay Hospital, The Bristol Royal Infirmary, and the University of Bristol for their assistance in identifying and preparing suitable material for the study.

Address for correspondence: Dr Robin Philipp, Department of Epidemiology and Community Medicine, University of Bristol, Canynge Hall, Whiteladies Road, Bristol BS8 2PR

\section{References}

${ }^{1}$ Mackie RM. The pathogenesis of cutaneous malignant melanoma. Br Med J 1983; 287: 1568-9.

${ }^{2}$ Magnus K. Incidence of malignant melanoma of the skin in Norway 1955-1970. Variations in time and space and

3 solar radiation. Cancer 1973; 32: $1275-86$.
${ }^{3}$ Green A. Incidence and reporting of cutaneous melanoma in Queensland. Australasian J Dermatol 1982; 23: 105-9. $\stackrel{\rho}{\rightarrow}$

${ }^{4}$ Cooke KR, Skegg DCG, Fraser J. Tends in malignant $\stackrel{2}{2}$ melanoma of skin in New Zealand. Int J Cancer 1983; 31: 715-8.

${ }^{5}$ Acheson ED. Mortality from cutaneous malignant melanoma. Health Trends 1986; 18: 73.

${ }^{6}$ Editorial. The aetiology of melanoma. The Lancet 1981; i 253-5.

7 Jones RE, Cash ME, Ackerman AB. Malignant melanoma mistaken histologically for junctional naevi. In: Pathology of malignant melanoma. Ed Ackerman AB. Masson Monographs in Dermatopathology, Vol 1. New York, 1981; 93-106.

${ }^{8}$ Editorial. Epidemiological approach to malignant melanoma. Br Med J 1972; 1: 130.

9 Editorial. More on melanoma. The Lancet 1985; i: 201-2.

${ }^{10}$ Swerdlow AJ. Incidence of malignant melanoma of the skin in England and Wales and its relationship to sunshine. Br Med J 1979; 2: 1324-7.

11 Bodenham DC. A study of 650 observed malignant melanomas in the South Western Region. Ann Roy Coll Surg Engl 1968; 43: 218-39.

${ }^{12}$ Griffiths RW, Briggs JC, Hiles RW. Clinical diagnostic accuracy in the management of primary stage 1 cutaneous malignant melanoma in a plastic surgery unit. Bristol Medico-Chirurgical J 1984; 99: 55-60.

${ }^{13}$ Philipp R, Hughes AO, Robertson MC, Mitchell TF. Malignant melanoma and association with arsenic. Bristol Medico-Chirurgical J 1983; 98: 165-9.

${ }^{14}$ Smith JHF, Al-Sam SZ, Briggs JC, Davies JD. An approach to histopathological audit in Bristol. Bristol Medico-Chirurgical J 1986; 101: 30-2.

${ }^{15}$ Mackie RM, Soutar DS, Watson ACH, McLaren KM, McPhie JL, Hutcheon AW, Smyth JF, et al. Malignant melanoma in Scotland 1979-1983. The Lancet 1985; ii: $859-62$.

${ }^{16}$ Gordon LG, Lowry WS. Incidence and aetiology of melanoma. The Lancet 1985; i: 583. 